\title{
Transport of comets to the outer planetary system
}

\author{
Arika Higuchi ${ }^{1,2}$ Eiichiro Kokubo ${ }^{1}$ \\ and Tadashi Mukai ${ }^{2}$ \\ ${ }^{1}$ Division of Theoretical Astronomy, National Astronomical Observatory of Japan, \\ 2-21-1 Osawa Mitaka Tokyo, Japan \\ email: higuchia@th.nao.ac.jp \\ ${ }^{2}$ Graduate School of Science and Technology, Kobe University \\ 1-1 Rokkodai-cho Nada Kobe Hyogo, Japan
}

\begin{abstract}
In the late stage of planet formation, planetesimals are perturbed by large (proto) planets. There are four fates of planetesimals, (1) to collide with planets, (2) to escape from the planetary region, (3) to survive in the planetary region, and (4) to fall onto the central star. The ratios of these fates depend on initial orbital parameters. We performed numerical simulations of gravitational scattering of planetesimals by a planet. We obtained the escape rate of planetesimals and its dependence on the orbital parameters of the planetesimals and the planet. We also calculated the rate for increasing the semimajor axis to more than 3000AU. Using these results, we discuss the relative efficiency of the four giant planets of the solar system in the formation of the Oort cloud.
\end{abstract}

Keywords. Oort cloud, comets: general, solar system: formation

\section{Introduction}

The Oort cloud is the spherical comet reservoir surrounding the solar system, as first proposed by Oort (1950). He suggested that the Oort cloud is constructed from planetesimals through two dynamical stages: (1) transportation of planetesimals to the outer region of the solar system by planets, and (2) pulling up of their perihelion distances by the external forces of the galactic tide and passing stars. The first dynamical stage was studied by several authors (e.g., Safronov 1972, Weidenschilling 1975). For example, Weidenschilling (1975) studied the ejection rate of planetesimals by the four giant planets analytically. He concluded that Jupiter ejected planetesimals with very high rate because of its large mass and it can not form the Oort cloud. Simulations of both of the first and the second stages were recently done by Dones et al. (2004). They showed that Saturn is the planet most responsible for the Oort cloud formation. They deal with the solar system and treat the two dynamical stages together. However, they do not focus on the general properties of the elementary processes of the planet-planetesimal scattering.

In this paper, we investigate the first dynamical stage of the comet cloud formation by using numerical calculations. On this first stage, planetesimals have four fates under the strong gravitational influence of a planet: (1) collision with the planet, (2) escape from the planetary system, (3) survival as a planetesimal, and (4) fall onto the central star. We investigate the dependence of the rates of these fates upon the orbital parameters. 
Table 1. The ranges of the initial parameters.

\begin{tabular}{cccccc}
\hline parameter & $a$ & $e$ & $i[\mathrm{rad}]$ & $a_{\mathrm{p}}[\mathrm{AU}]$ & $m_{\mathrm{p}}\left[m_{\mathrm{J}}\right]$ \\
\hline range & $\frac{a_{\mathrm{p}}}{1+e} \lesssim a \lesssim \frac{a_{\mathrm{p}}}{1-e}$ & $0-0.9$ & $0.01-0.1$ & $1,5,10,30$ & $0.1-10$ \\
\hline
\end{tabular}

\section{Method of Calculation}

\subsection{Model and Integration Method}

We integrate the orbits of planetesimals numerically using the forth-order Hermite scheme (Makino \& Aarseth 1992) with the hierarchical timestep (Makino 1991). We assume a planet around a central star on a circular orbit and a mass-less planetesimal orbiting the central star under the gravity of the planet. The equation of motion of the planetesimal is

$$
\frac{d^{2} \mathbf{r}}{d t^{2}}=-G m_{\star} \frac{\mathbf{r}}{r^{3}}-G m_{\mathrm{p}}\left(\frac{\mathbf{r}-\mathbf{r}_{\mathrm{p}}}{\left|\mathbf{r}-\mathbf{r}_{\mathrm{p}}\right|^{3}}+\frac{\mathbf{r}_{\mathrm{p}}}{r_{\mathrm{p}}^{3}}\right),
$$

where $r$ is the heliocentric distance of the planetesimal, $G$ is the gravitational constant, $m_{\star}$ is the mass of the central star, and $m_{\mathrm{p}}$ and $r_{\mathrm{p}}$ are the mass and the heliocentric distance of the planet. The last term on the r.h.s represents the indirect term.

\subsection{Initial Conditions and Parameters}

We assume an initial planetesimal disk which consists of mass-less planetesimals and a planet. All the planetesimals have the same eccentricity $e$ and inclination $i$. The inner and outer edges, $a_{\min }$ and $a_{\max }$, of the disk are $a_{\min }=a_{\mathrm{p}} /(1+e), a_{\max }=a_{\mathrm{p}} /(1-e)$, respectively. Here $a_{\mathrm{p}}$ is the semimajor axis of the planet. The argument of perihelion $\omega$ and the longitude of ascending node $\Omega$ are distributed randomly. The number density of planetesimals is proportional to $a^{-1}$. Our model contains $10^{7}$ planetesimals per ring with the width of $1 \mathrm{AU}$. The ranges of the initial parameters are shown in Table.1. We consider the parameter set $i=0.05 \mathrm{rad}, a_{\mathrm{p}}=5 \mathrm{AU}$, and $m_{\mathrm{p}}=m_{\mathrm{J}}$ as the standard case. We define $m_{\mathrm{J}} \equiv 0.001 m_{\star}$ where we set $m_{\star}=m_{\odot}$ (solar mass). In all cases, we calculate orbits of planetesimals for 1 Kepler period $\left(T_{\mathrm{K}}\right)$. During the orbit integration, if the separation between a planet and a planetesimal becomes smaller than the radius of the planet $R_{\mathrm{p}}$, or the heliocentric distance of a planetesimal becomes smaller than the radius of a central star $R_{\star}$, the planetesimal is counted as "collider" or "fall", respectively. Planetesimals not yet classified as colliders and falls are checked for their orbital elements at the final time $T_{\mathrm{K}}$. If the perihelion distance of a planetesimal is smaller than $R_{\star}$, it is also counted as "fall". If the eccentricity of a planetesimal is larger than 1 , it is counted as "escaper". Following to Dones et al. (2004), here we assume that planetesimals with $a>3000 \mathrm{AU}$ or $e>1$ can go to the next dynamical stage. Thus a planetesimal with $a>3000 \mathrm{AU}$ and $e<1$ is counted as "candidate", candidate for a member of the Oort cloud.

\subsection{Definitions of Probability and Efficiency}

We denote $P_{\text {esc }}$ and $P_{\text {can }}$ the probabilities of escape and candidate after a time span $T_{\mathrm{K}}$. Using these probabilities $P$, we define the efficiencies $K$. Efficiencies $K_{\text {esc }}$ and $K_{\text {can }}$ mean the expected numbers of escaper and candidate per time and are defined as

$$
K=\int_{a_{\min }}^{a_{\max }} P T_{\mathrm{K}}^{-1} n_{\mathrm{s}}(a) 2 \pi a d a,
$$

where $n_{\mathrm{S}}(a)$ is a surface number density of planetesimals estimated as

$$
n_{\mathrm{s}}(a)=n_{0} a^{\theta}
$$


where $n_{0}$ is the surface number density at $a=1 \mathrm{AU}$, and $\theta$ is the power-index of the distribution.

\section{Probabilities}

\subsection{Escape from the Planetary System}

Figure 1 shows $P_{\text {esc }}$ against $a$, with $e=0.8$ and $e=0.7$ for the standard case. The orbitcrossing regions with these parameters are from $2.78 \mathrm{AU}$ to $25 \mathrm{AU}$ and from $2.94 \mathrm{AU}$ to 16.7AU, respectively. Escapers appear almost over the entire orbit-crossing region. In this region, $P_{\text {esc }}$ increases along $a$ and suddenly drops around the end of the region. The orbitcrossing region increase with $e . P_{\mathrm{esc}}$ decreases with $e$ at fixed $a$. There are no escapers

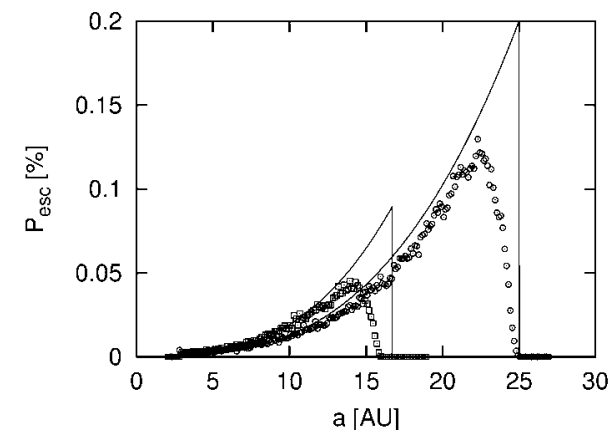

Figure 1. Probabilities of escape and the empirical fits are plotted against $a$. The circles and squares indicate $P_{\text {esc }}$ with $e=0.8$ and $e=0.7$ for the standard case $\left(i, a_{\mathrm{p}}, m_{\mathrm{p}}\right)=(0.05,5,1)$.

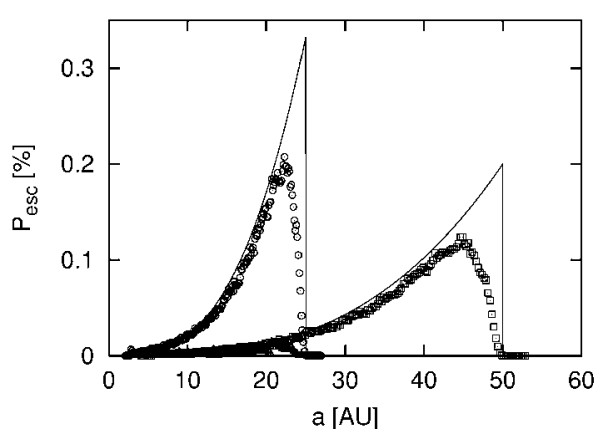

Figure 2. The same figure as figure 1 . The symbols indicate $P_{\text {esc }}$ for $e=0.8,\left(i, a_{\mathrm{p}}, m_{\mathrm{p}}\right)=$ $(0.03,5,1)$ (circles), $(0.05,10,1)$ (squares), and $(0.05,5,0.3)$ (triangles), respectively.

for $e<0.4$. This absence of escaper is explained by the relative velocity $v_{\mathrm{r}}$ between a planetesimal and a planet and the fly-by theory under the two-body approximation. Using the conservation of the Jacobi energy, $v_{\mathrm{r}}$ is written as $v_{\mathrm{r}}^{2}=\left\{3-2\left[\left(1-e^{2}\right) a / a_{\mathrm{p}}\right]^{1 / 2} \cos i-\right.$ $\left.a_{\mathrm{p}} / a\right\} v_{\mathrm{p}}^{2}$, where $v_{\mathrm{p}}$ is the Kepler velocity of the planet. To escape from the planetary system, $v_{\mathrm{r}}$ need to satisfy $\left|\mathbf{v}_{\mathrm{p}}+\mathbf{v}_{\mathrm{r}}\right|>v_{\text {parabolic }}$, where $v_{\text {parabolic }}$ is the local escape velocity written as $\sqrt{2 G m_{\star} / a_{\mathrm{p}}}$. There is a minimum relative velocity $v_{\mathrm{r}}^{\text {min }}$ to escape derived from the fly-by theory. A planetesimal gains the largest additional velocity of $v_{\mathrm{r}}$, if it is scattered toward the direction of the velocity vector of the planet. Here $v_{\mathrm{r}}^{\min }$ is given by

$$
\begin{aligned}
v_{\mathrm{r}}^{\min } & =v_{\text {parabolic }}-v_{\mathrm{p}} \\
& =(\sqrt{2}-1) v_{\mathrm{p}} .
\end{aligned}
$$

To satisfy the condition $v_{\mathrm{r}}>v_{\mathrm{r}}^{\mathrm{min}}$, we need $e \gtrsim 0.4$. So planetesimals initially with $e \lesssim 0.4$ can not escape. Figure 2 is the same as figure 1 but with different $i, a_{\mathrm{p}}$, and $m_{\mathrm{p}}$. $P_{\text {esc }}$ decreases with $i$. The orbit-crossing region increases with $a_{\mathrm{p}}$ and $P_{\mathrm{esc}}$ at fixed $a$ decreases with $a_{\mathrm{p}}$ but they can be scaled by $a_{\mathrm{p}}$. This is because $v_{\mathrm{r}}$ is scaled by $a_{\mathrm{p}} . P_{\mathrm{esc}}$ increases with $m_{\mathrm{p}}$. This is because the cross section for strong scattering is proportional to $m_{\mathrm{p}}^{2}$. The main features of $P_{\text {esc }}$ do not change when these parameters are changed. We 
plot the empirical fit with the approximated dependences, given by

$$
P_{\mathrm{esc}}^{\mathrm{fit}} \sim 4\left(\frac{a}{a_{\mathrm{p}}}\right)^{3}(1-e) \sin ^{-1} i m_{\mathrm{p}}^{2},
$$

in figures 1 and 2 . Here we set the unit mass as $m_{\star}$. The derivation of this expression is discussed in another paper.

\subsection{Candidate for the Oort Cloud}

Figure 3 shows $P_{\text {can }}$ against $a$, with $e=0.8$ for the standard case. Candidates appear almost over the orbit-crossing region. In this region, $P_{\text {can }}$ increases along $a$ and suddenly drops around the end of the region. This behavior is similar to that of $P_{\text {esc }}$. However, the dependence on $a$ is different. Figure 3 also shows $P_{\text {can }}$ with $e=0.7$. Figure 4 is the same as figure 3 but with different $i, a_{\mathrm{p}}$, and $m_{\mathrm{p}}$. The dependences of $P_{\text {can }}$ on $i$ and $m_{\mathrm{p}}$ are the same as those of $P_{\text {esc }}$. The main features of $P_{\text {can }}$ do not change when these parameters are changed. In figures 3 and 4 , we plot the empirical fit given by

$$
P_{\mathrm{can}}^{\mathrm{fit}} \sim 12\left(\frac{a_{\mathrm{can}}}{a_{\mathrm{p}}}\right)^{-1}\left(\frac{a}{a_{\mathrm{p}}}\right)^{5}(1-e)^{2} \sin ^{-1} i m_{\mathrm{p}}^{2} .
$$

This expression is valid under two conditions: (1) $m_{\mathrm{p}} \lesssim 3 m_{\mathrm{J}}$ and (2) $a_{\text {can } /} a_{\mathrm{p}} \gtrsim 100$. The derivation of this expression is also discussed in another paper.

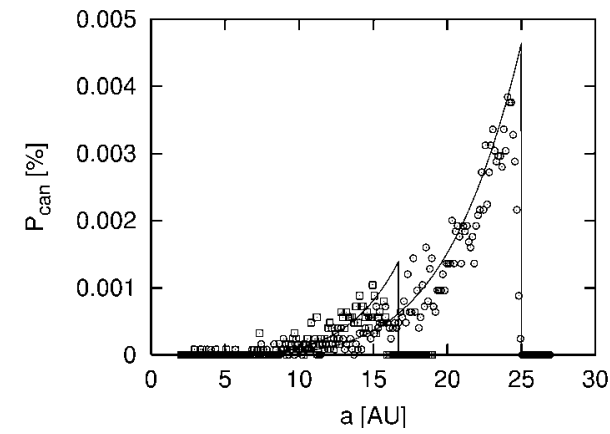

Figure 3. Probabilities of candidate and the empirical fits are plotted against $a$. Symbols and parameters are the same as figure 1.

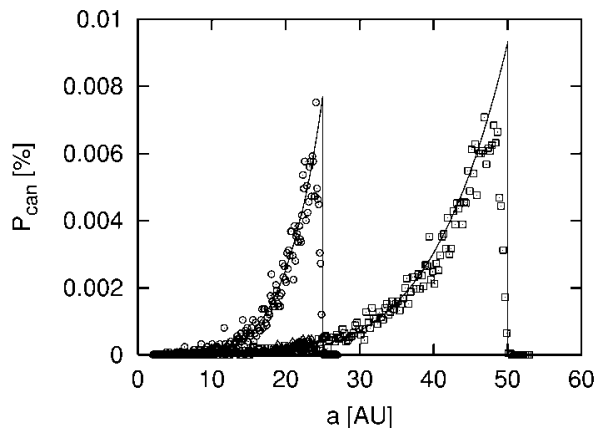

Figure 4. The same figure as figure 2. Symbols and parameters are also the same as figure 2 .

\section{Efficiencies}

Using the probabilities of $P_{\text {esc }}$ and $P_{\text {can }}$, we obtain the efficiencies of escape $K_{\text {esc }}$ and candidate $K_{\text {can }}$. Here we adopt the standard disk model of $\theta=-3 / 2$ (Hayashi 1981) and $n_{0}=1$ for simplicity. The integration is performed over the orbit-crossing region. Figures 5 and 6 show the dependence of $K_{\text {esc }}$ and $K_{\text {can }}$ on $e$. Both $K_{\text {esc }}$ and $K_{\text {can }}$ increase with $e$ for $e>0.4$. From the dependences of $P_{\mathrm{esc}}^{\mathrm{fit}}, P_{\mathrm{can}}^{\mathrm{fit}},(2.2)$, and the disk model we assumed, we obtain the dependences $K_{\mathrm{esc}}^{\mathrm{fit}} \propto \sin ^{-1} i a_{\mathrm{p}}^{-1} m_{\mathrm{p}}^{-2}$ and $K_{\mathrm{esc}}^{\mathrm{fit}} \propto$ $\sin ^{-1} i a_{\text {can }}^{-1} m_{\mathrm{p}}^{-2}$. For $e \gtrsim 0.5$ and $i<0.07, K_{\text {esc }}^{\text {fit }}$ agrees $K_{\text {esc }}$ within a factor of $\sim 2$ and $K_{\text {can }}^{\text {fit }}$ agrees $K_{\text {can }}$ within a factor of $\sim 4$. 


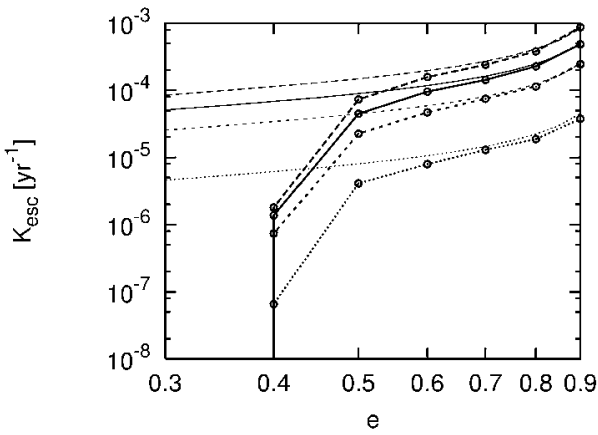

Figure 5. Efficiencies of escape(symbol) and the empirical fits are plotted against $e$. The solid, dash, short-dash, and dotted lines indicate $K_{\text {esc }}$ for $\left(i, a_{\mathrm{p}}, m_{\mathrm{p}}\right)=(0.05,5,1)$ (standard case $),(0.03,5,1),(0.05,10,1)$, and $(0.05,5,0.3)$, respectively.

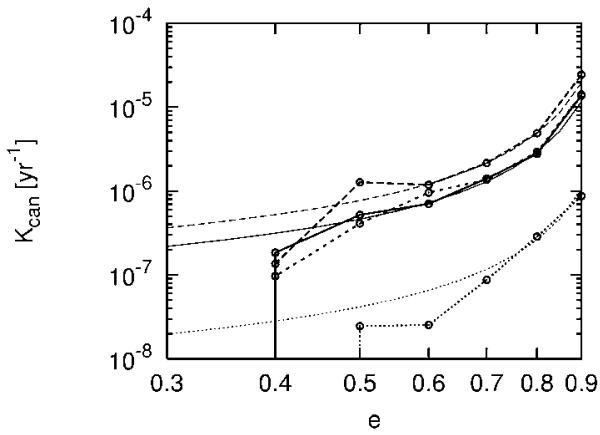

Figure 6. Efficiencies of candidate and the empirical fits are plotted against $e$. Lines and parameters are the same as in figure 5 .

\section{Application to the Oort Cloud Formation}

We apply the results to the Oort cloud formation. We assume that the planetesimal disk has the outer edge at 50AU and adopt the four giant planets with present $a_{\mathrm{p}}$ and $m_{\mathrm{p}}$.

\subsection{Standard Disk}

Figure 7 shows $K_{\text {esc }}$ and $K_{\text {can }}$ with $i=0.05 \mathrm{rad}$ and $n_{\mathrm{s}}=a^{-3 / 2}$. For Saturn, Uranus, and Neptune, $K_{\mathrm{esc}}$ and $K_{\mathrm{can}}$ decrease with $e$ for $e>0.8, e>0.6$, and $e>0.5$ respectively. This is because of the finite disk extent. Jupiter has the highest $K_{\text {esc }}$ and $K_{\text {can }}$. All planets have $K_{\text {esc }}$ higher than $K_{\text {can }}$. This means that Jupiter is the planet most responsible for transporting planetesimals to the Oort cloud, even if it produces many escapers. The roles of Uranus and Neptune are comparable and both are much less than that of the other two planets.

\subsection{Hot Disk}

We vary $i$ of planetesimals around each planet. The massive planet effectively excites the eccentricity and inclination of nearby planetesimals. Here we adopt $i=\left(m_{\mathrm{p}} / 3 m_{\star}\right)^{1 / 3}$, the reduced Hill radius of the planet. Figure 8 shows $K_{\text {can }}$ with $i=\left(m_{\mathrm{p}} / 3 m_{\star}\right)^{1 / 3}$ and $n_{\mathrm{s}}=a^{-3 / 2}$. In this case, the planetesimals around Jupiter have the highest $i$ and this reduces $K_{\text {can }}$. However, Jupiter still has the highest value and the relationships among the efficiencies of the four planets are not changed.

\section{Summary and Discussion}

We investigated the orbital evolution of planetesimals scattered by a planet using numerical calculations. We obtained the probabilities and the efficiencies in producing escapers and candidates and their empirical expressions. We applied these results to the Oort cloud formation. We consider two disk models: (1) standard disk and (2) hot disk. With both disk models, Jupiter is the most effective planet to produce escape and candidate for the Oort cloud. However, we have to consider more realistic initial condition, especially the orbital elements of planetesimals around a planet. They depend on the parameter of the planet, the existence of the gas disk, and the interaction among planetesimals. We will investigate this in the near future work. 


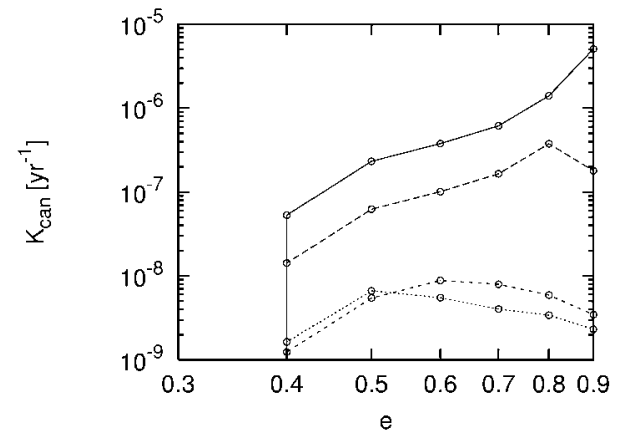

Figure 7. Efficiencies $K_{\text {esc }}$ and $K_{\text {can }}($ symbol) with the standard disk, $\mathrm{i}=0.05 \mathrm{rad}, n_{\mathrm{s}}=a^{-3 / 2}$ are plotted against $e$. The solid, dash, short-dash, and dotted lines indicate Jupiter, Saturn, Uranus, and Neptune, respectively.

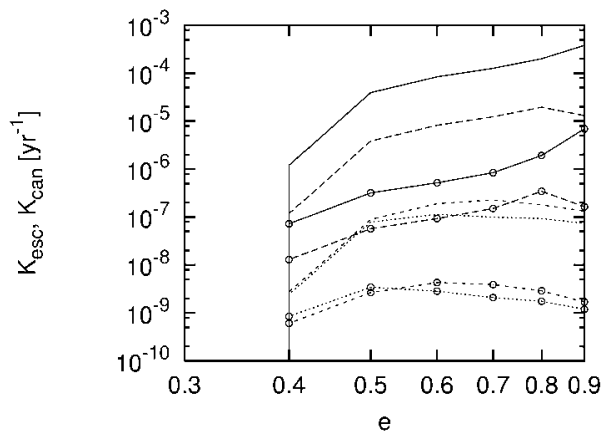

Figure 8. The same figure as 7 but with the hot disk, $i=\left(m_{\mathrm{p}} / 3 m_{\odot}\right)^{1 / 3}$, and $n_{\mathrm{s}}=a^{-3 / 2}$. Only $K_{\text {can }}$ is plotted.

Our calculations deal with the elementary process of gravitational scattering of planetesimals by a planet. The parameter ranges we used are not restricted to the solar system. Thus, we can apply the results to the extrasolar planetary systems. Using these results, we can also estimate the formation rate of the comet clouds around the extrasolar planetary systems.

\section{Acknowledgements}

This work was supported by the 21st Century COE Program Origin and Evolution of Planetary Systems of the Ministry of Education, Culture, Sports, Science, and Technology, Japan, and JSPS Research Fellowship for Young Scientists.

\section{References}

Duncan, M., Quinn, T. \& Tremaine, S. 1987, Astron. J. 94, 1330

Dones, L., Levison, H.F., Duncan, M. \& Weissman, P. 2004, Icarus, in press

Makino, J 1991, Publ. Astron. Soc. Japan 43, 859

Makino, J. \& Aarseth, S.J. 1992, Publ. Astron. Soc. Japan 44, 141

Hayashi, C 1981, Prog. Theor. Phys. Suppl. 70, 35

Oort, J.H 1950, Bull. Astron. Inst. Neth. 11, 91

Safronov, V.S. 1972, in: G. A. Chebotarev, E. I. Kazimirchak-Polonskaia \& B. G. Marsden (eds.), Proceedings IAU Symp.45: The Motion, Evolution of Orbits, and Origin of Comets, (Dordrecht: Reidel), p. 329

Weidenschilling, S.J. 1975, Astron. J. 80, 145 